University of Nebraska - Lincoln

DigitalCommons@University of Nebraska - Lincoln

John A. Woollam Publications

Electrical \& Computer Engineering, Department

\title{
Vacuum Ultraviolet Optical Analysis of Spin-Cast Chitosan Films Modified by Succinic Anhydride and Glycidyl Phenyl Ether
}

\author{
W. H. Nosal \\ University of Nebraska - Lincoln \\ D. W. Thompson \\ University of Nebraska-Lincoln, dthompson2@unl.edu \\ T. E. Tiwald \\ J. A. Woollam Co. \\ S. Sarkar \\ University of Nebraska - Lincoln \\ Anuradha Subramanian \\ University of Nebraska - Lincoln, asubramanian2@unl.edu \\ See next page for additional authors
}

Follow this and additional works at: https://digitalcommons.unl.edu/elecengwoollam

Part of the Electrical and Computer Engineering Commons

Nosal, W. H.; Thompson, D. W.; Tiwald, T. E.; Sarkar, S.; Subramanian, Anuradha; and Woollam, John A., "Vacuum Ultraviolet Optical Analysis of Spin-Cast Chitosan Films Modified by Succinic Anhydride and Glycidyl Phenyl Ether" (2007). John A. Woollam Publications. 1.

https://digitalcommons.unl.edu/elecengwoollam/1

This Article is brought to you for free and open access by the Electrical \& Computer Engineering, Department of at DigitalCommons@University of Nebraska - Lincoln. It has been accepted for inclusion in John A. Woollam Publications by an authorized administrator of DigitalCommons@University of Nebraska - Lincoln. 


\section{Authors}

W. H. Nosal, D. W. Thompson, T. E. Tiwald, S. Sarkar, Anuradha Subramanian, and John A. Woollam 


\title{
Vacuum ultraviolet optical analysis of spin-cast chitosan films modified by succinic anhydride and glycidyl phenyl ether
}

\author{
W. H. Nosal, ${ }^{1}$ D. W. Thompson, ${ }^{1}$ T. E. Tiwald, ${ }^{3}$ S. Sarkar, ${ }^{2}$ A. Subramanian,,${ }^{2}$ and J. A. Woollam ${ }^{1 *}$ \\ ${ }^{1}$ Department of Electrical Engineering, University of Nebraska-Lincoln, Lincoln, NE 68588 USA \\ ${ }^{2}$ Department of Chemical Engineering, University of Nebraska-Lincoln, Lincoln, NE 68588 USA \\ ${ }^{3}$ J. A. Woollam Co., 645 M Street, Suite 102, Lincoln, NE 68508, USA \\ * Corresponding author: J. A. Woollam, Department of Electrical Engineering, \\ University of Nebraska-Lincoln, Lincoln, NE 68588, USA. Email: jwoollam@jawoollam.com
}

\begin{abstract}
Optical properties of spin-cast chitosan films were determined in the vacuum ultraviolet (VUV) through visible regions of the spectrum using spectroscopic ellipsometry. The onset of absorption in the ultraviolet was determined for chitosan films modified with succinic anhydride (SA) and glycidyl phenyl ether (GPE). This absorption was accounted for by including Gaussian and TaucLorentz shaped oscillators in the optical model. VUV through visible optical constants were determined for these modified films, as well as for immunoglobulin G (IgG) attached to GPE-modified samples. Several resonant oscillator structures exhibit greatly differing oscillator parameters for the as-deposited, as well as SA-and GPE-modified films. These in-plane and out-of-plane oscillator strengths, energy positions, and broadening were determined and tabulated, and their chemical origins identified. A phenyl resonant p-bond at approximately $6.3 \mathrm{eV}$ was observed for the GPE-modified sample. This was not present in the bare chitosan data. GPE contains a phenyl group in the molecule and absorption due to it is clearly identified. Clarity of data after surface chemical modification is due to the high surface sensitivity of VUV ellipsometry.
\end{abstract}

Keywords: chitosan, ellipsometry, optical constants, spectroscopy, vacuum ultraviolet

\section{INTRODUCTION}

Chitin is a carbohydrate biopolymer and a major component in shells of crustaceans such as shrimp and crabs. ${ }^{1}$ Chitin consists of repeated units of $\mathrm{N}$-acetyl-D-glucosamine joined together by $\beta(1 \rightarrow 4)$ linkages. Chitosan is a chemically modified form of chitin, where, through hydrolysis, the acetyl group attached to the nitrogen atom is replaced by hydrogen, yielding a primary amine group. Both chitin chitosan have a chemical structure similar to cellulose. The structures ${ }^{1}$ of chitin, chitosan, and cellulose are shown in Figure 1.

\section{EXPERIMENTAL}

\section{Sample preparation}

For this study, thin films of chitosan were produced by spincasting a solution of chitosan onto (100) orientated silicon wafers. Wafers were $50 \mathrm{~mm}$ in diameter, $0.4 \mathrm{~mm}$ thick, polished on one side, and typically had $1.6 \mathrm{~nm}$ of native oxide.

Chitosan is soluble in acidic conditions. Solutions used to produce films were $2 \%$ weight to volume $(\mathrm{w} / \mathrm{v})$ of chitosan in acetic acid solvent. This acetic acid solvent was a $2 \% \mathrm{w} / \mathrm{v}$ solution of acetic acid in deionized water. Powdered chitosan and the acetic acid solution were mixed at room temperature on a stir plate for $18 \mathrm{~h}$, yielding a viscous liquid with a faint brown color, with viscosity of around 825 centipoise $(\mathrm{cP})$.

Initially, chitosan solutions yielded films with numerous small spots and comet defects due to small impurity particles. These particles caused spot defects through capillary action, creating areas of greater film thickness around the particle. Because of high solution viscosity and small particle size, an ultracentrifuge was required to settle these particles out of the solution. Centrifugation of solutions for $5 \mathrm{~h}$ with $27,500 \mathrm{~g}$ acceleration yielded defect-free films. ${ }^{2,3}$

After centrifugation, the samples were spin-coated onto 50 $\mathrm{mm}$ diameter silicon wafers at a speed of $2500 \mathrm{rpm}$ for $30 \mathrm{~s}$. By preloading the centrifuged solution and using the slowest acceleration ramp factor, comets and other typical spin-coat defects were eliminated or greatly reduced.

After spinning, the sample was removed and allowed to dry in air. Samples were then placed in a drying oven at $80^{\circ} \mathrm{C}$ for $4 \mathrm{~h}$ to increase adhesion of chitosan to the wafer. The acidity of the chitosan film was neutralized by immersion in $0.25 \mathrm{M}$ $\mathrm{NaOH}$ solution for $15 \mathrm{~min}$ followed by immersion in deionized 

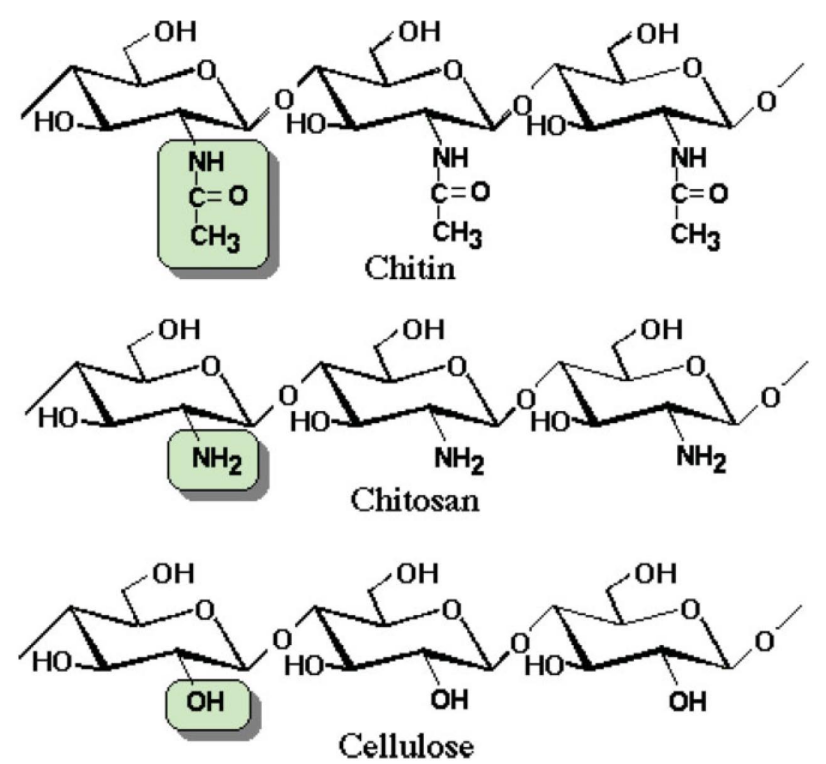

Figure 1. Structures of chitin and chitosan and their comparison to cellulose. (Reference 1).

water for $15 \mathrm{~min}$. The sample was gently blown dry and placed in a desiccator overnight to dry. The aforementioned process yields chitosan films of $320 \mathrm{~nm}$ thickness.

\section{Modifications}

Bare spin-cast chitosan films were modified to change the surface chemistry. ${ }^{4}$ One modification was the attachment of 1,2 epoxy-3-phenoxy-propane or glycidyl phenyl ether (GPE) to the film surface. The structure of GPE is given in Figure 2. The other surface modification was to attach succinic anhydride (SA) to the film surface. The structure of SA is given in Figure 3. Without further independent experiments it is not known how much of the inner regions of the film the modifications reach. Thus the modifications are assumed to be attached to the outer surface of the chitosan films (including its roughness). These modified films were then allowed to contact a solution of $1 \mu \mathrm{g} / \mathrm{ml}$ of immunoglobulin $\mathrm{G}$ (IgG) protein, and the uptake of protein was studied by ex situ vacuum ultraviolet (VUV) ellipsometry.

\section{VUV Ellipsometry}

An emerging ellipsometry technique is VUV-variable angle spectroscopic ellipsometry (VASE)..$^{5}$ In 1999 commercial VUV ellipsometers were introduced in response to the material char-

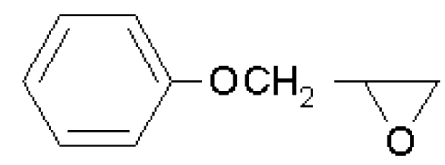

Figure 2. Chemical structure of glycidyl phenyl ether.<smiles>O=C1CCC(=O)O1</smiles>

Figure 3. Chemical structure of succinic anhydride. acterization requirements for $157 \mathrm{~nm}$ lithography, and cover the range 135-1700 nm (0.73-9.1 eV). VUV photon energies are useful to study high-energy electronic transitions, where the optical response is dominated by band-to-band transitions.

\section{Optical anisotropy}

In transparent materials where the dielectric permittivity depends on the direction of the electric field in the material, important anisotropic optical effects can be observed. ${ }^{6-8}$ This is typically measured as a difference in the indices of refraction, $n_{1}, n_{2}, n_{3}$. For example,

$$
\Delta n_{21}=n_{2}-n_{1}
$$

where $\Delta \mathrm{n}$ is the birefringence. Dichroism occurs when the extinction coefficient $\mathrm{k}$ is anisotropic.

In the spin-cast chitosan, the in-plane and out-of-plane indices of refraction were different, but uniform within the plane, thereby resulting in uniaxially anisotropy. Generalized ellipsometry analysis was used to analyze the films. ${ }^{8}$ Uniaxial anisotropy observed for spin-cast chitosan films is most likely a result of long polymer chains lying within the sample plane.

\section{DATA ANALYSIS}

An ellipsometer yields two measurable quantities (describing polarization changes) at each wavelength and angle of incidence, often expressed as $\Psi$ and $\Delta$. To determine physical parameters, an optical-model regression-based data analysis is performed. Numerous material parameters can be extracted, including layer thickness, surface and/or interfacial roughness, optical constants, and void fraction. ${ }^{9-11}$ In this work, data were analyzed assuming that the films were dense, smooth, and homogeneous, but uniaxially anisotropic.

Thickness and optical constants were determined from $\Psi$ and $\Delta$ data using a regression analysis that modifies the parameters until a best fit to data is obtained. For chitosan this was done in two steps. First the thickness of each film was determined by modeling data from the nonabsorbing region $(0.73-5.0 \mathrm{eV})$ using a Cauchy dispersion model including uniaxial anisotropy. ${ }^{12}$ Thickness was then fixed at this value and a uniaxial oscillator layer was used to model the absorbing region. In this layer, Gaussian and Tauc-Lorentz oscillators were added to account for resonance-like absorption, but Gaussian shapes dominated. ${ }^{13-15}$ With a known thickness, the dielectric function (equivalently, index of refraction and extinction coefficient) was determined over the entire range, absorbing or not. ${ }^{16}$

Note that from the dielectric function the absorption coefficient and penetration depth $\delta$ can be determined, which is the depth at which the intensity drops by $\mathrm{e}^{-1}$, not the depth at which light no longer penetrates. ${ }^{9}, 10,12$

\section{RESULTS}

The optical constants were determined from 0.73 to $9.1 \mathrm{eV}$. Data are plotted against photon energy (rather than wavelength) to better visualize features in the VUV region. At a $\mathrm{pH}$ of 7.4 
both the SA-modified surface and the IgG molecule have a net negative charge and therefore no attachment should occur.

As a case study, three samples were examined using vacuum ultraviolet spectroscopic ellipsometry (VUV-SE): a bare chitosan thin film, a GPE-modified chitosan film, and an SA-modified chitosan thin film. The optical constants of chitosan are presented in the form of the real and imaginary dielectric functions $\varepsilon_{1}$ and $\varepsilon_{2}$ in Figure 4. Figure 5 shows the dielectric function for GPE-modified chitosan. In Figure 6, the dielectric functions are presented for SA-modified chitosan.

Gaussian oscillator parameters for unmodified chitosan in the VUV region for both in-plane and out-of-plane oscillators

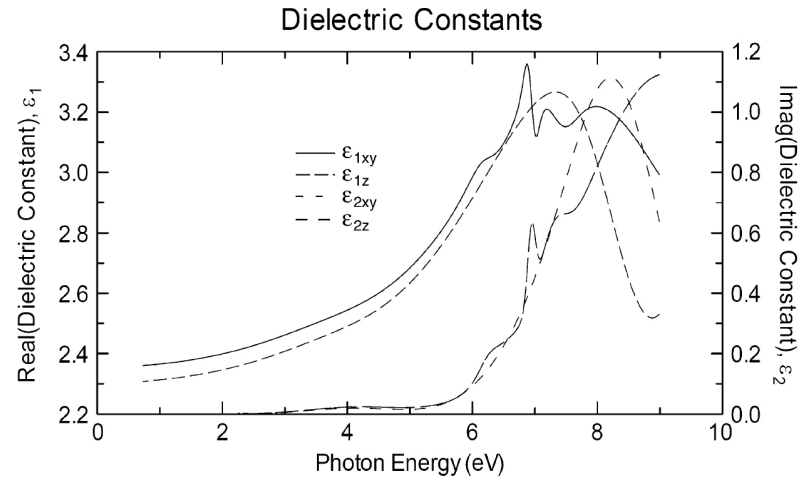

Figure 4. Real and imaginary dielectric functions for unmodified chitosan.

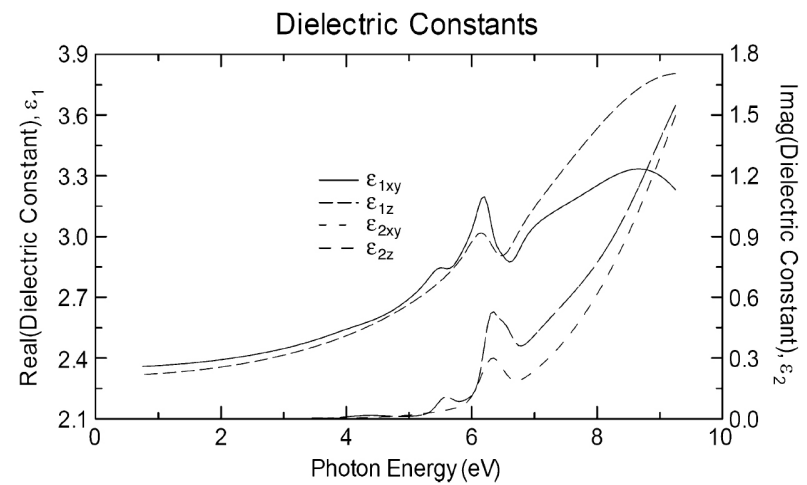

Figure 5. Real and imaginary dielectric functions for GPE-modified chitosan.

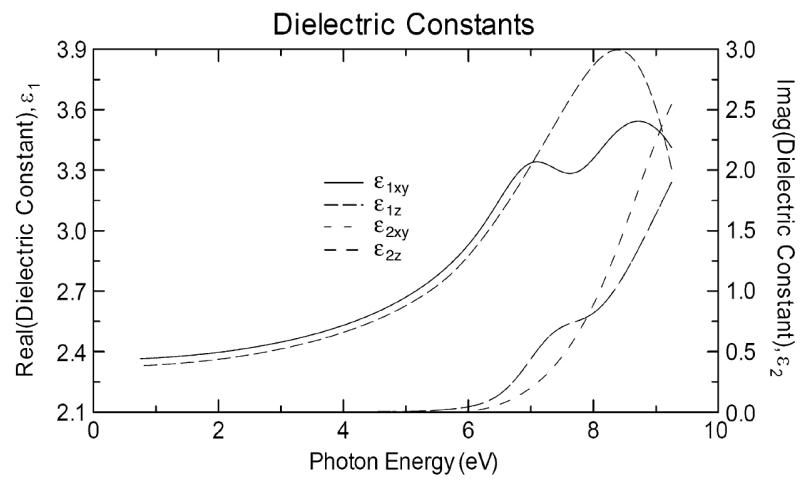

Figure 6. Real and imaginary dielectric functions for succinic anhydride modified chitosan. are given in Table 1. Those for GPE-modified chitosan are seen in Table 2, and for IgG attached to GPE-modified films in Table 3 . Note the rather small changes in oscillator parameters upon IgG attachment.

The SA-modified film was modeled using two in-plane Gaussian oscillators and one out-of-plane Tauc-Lorentz oscillator. The lack of phenyl groups in the SA modification is the likely reason for fewer oscillators required in the model. Tables 4 and 5 show the oscillator parameters.

Figure 7 allows comparison of the in-plane $\varepsilon_{1}$ for all three samples as a function of photon energy. Figure 8 shows the inplane $\varepsilon_{2}$ for these three samples as a function of photon energy.

In all samples several noticeable chemistries are seen in the VUV data. Detectable chromophores are the carbonyl $\pi$-bond at approximately $7.3 \mathrm{eV}$, the $\mathrm{C}-\mathrm{O} \sigma$-bond at approximately 7.0 $\mathrm{eV}$, the $\mathrm{C}-\mathrm{N} \sigma$-bond at approximately $6.5 \mathrm{eV}$, the $\mathrm{C}-\mathrm{C} \sigma$-bond at approximately $9.1 \mathrm{eV}$, and the phenyl resonant $\pi$-bond at approximately $6.3 \mathrm{eV} \cdot{ }^{17}$ It is of utmost importance to point out the feature at approximately $6.3 \mathrm{eV}$ for the GPE-modified sample. GPE contains a phenyl group in the molecule and absorption due to it is clearly seen in the data.

Because it is only the GPE-modified chitosan that contains a phenyl group, this feature is present only in the curve of

Table 1. Gaussian oscillator parameters for unmodified chitosan in the VUV region. Note that in-plane oscillator 4 is Tauc-Lorentz type.

\begin{tabular}{lccll}
\hline $\begin{array}{l}\text { In-plane } \\
\text { oscillators }(x y)\end{array}$ & Amplitude & $\begin{array}{c}\text { Centering } \\
\text { energy }(\mathrm{eV})\end{array}$ & Broadening $(\mathrm{eV})$ \\
\hline 1 & 1.18 & 9.08 & 3.15 & \\
2 & 0.154 & 7.28 & 0.488 & \\
3 & 0.244 & 6.95 & 0.147 & \\
4 & 3.40 & 6.21 & $c=0.569 \quad E_{\mathrm{g}}=5.54$ \\
5 & .0227 & 4.12 & 1.71 & \\
Out-of-plane & & & & \\
oscillators $(z)$ & & & & \\
1 & 0.699 & 8.37 & 1.54 & \\
2 & 0.503 & 7.71 & 2.18 & \\
3 & 0.0194 & 4.10 & 1.65 & \\
\hline
\end{tabular}

Table 2. Gaussian oscillator parameters for GPE-modified chitosan in the VUV region.

\begin{tabular}{lccc}
\hline $\begin{array}{l}\text { In-plane } \\
\text { oscillators }(x y)\end{array}$ & Amplitude & $\begin{array}{c}\text { Centering } \\
\text { energy }(\mathrm{eV})\end{array}$ & Broadening $(\mathrm{eV})$ \\
\hline 1 & 1.87 & 10.14 & 3.32 \\
2 & 0.268 & 7.26 & 1.97 \\
3 & 0.214 & 6.49 & 0.321 \\
4 & 0.256 & 6.28 & 0.228 \\
5 & 0.0627 & 5.57 & 0.284 \\
6 & 0.0160 & 4.35 & 0.864 \\
Out-of-plane & & & \\
oscillators $(z)$ & & & \\
1 & 3.22 & 11.91 & 5.06 \\
2 & 0.189 & 6.31 & 0.414 \\
\hline
\end{tabular}


Table 3. Gaussian oscillator parameters for GPE-modified chitosan after IgG attachment, in the VUV region.

\begin{tabular}{lccc}
$\begin{array}{l}\text { In-plane } \\
\text { oscillators }(x y)\end{array}$ & Amplitude & $\begin{array}{c}\text { Centering } \\
\text { energy }(\mathrm{eV})\end{array}$ & Broadening $(\mathrm{eV})$ \\
\hline 1 & 2.00 & 10.37 & 3.36 \\
2 & 0.287 & 7.61 & 2.02 \\
3 & 0.268 & 6.42 & 0.567 \\
4 & 0.160 & 6.30 & 0.214 \\
5 & 0.0918 & 5.60 & 0.363 \\
6 & 0.0170 & 4.44 & 0.943 \\
Out-of-plane & & & \\
oscillators $(z)$ & & & 5.11 \\
1 & 3.32 & 12.04 & 0.380 \\
2 & 0.290 & 6.41 & \\
\hline
\end{tabular}

Table 4. Gaussian oscillator parameters for SA-modified chitosan films in the VUV region.

\begin{tabular}{lccc}
\hline $\begin{array}{l}\text { In-plane } \\
\text { oscillators }(x y)\end{array}$ & Amplitude & $\begin{array}{c}\text { Centering } \\
\text { energy }(\mathrm{eV})\end{array}$ & Broadening $(\mathrm{eV})$ \\
\hline 1 & 2.78 & 10.65 & 3.78 \\
2 & 0.318 & 7.35 & 1.04 \\
\hline
\end{tabular}

Table 5. Tauc-Lorentz oscillator parameters for SA-modified chitosan film.

\begin{tabular}{lcccc}
\hline $\begin{array}{l}\text { Out-of-plane } \\
\text { oscillator }(z)\end{array}$ & Amplitude & $\begin{array}{c}\text { Centering } \\
\text { energy }(\mathrm{eV})\end{array}$ & $\begin{array}{c}\mathrm{C} \\
(\mathrm{eV})\end{array}$ & $\begin{array}{c}E_{\mathrm{g}} \\
(\mathrm{eV})\end{array}$ \\
\hline 1 & 62.03 & 9.50 & 3.53 & 5.72 \\
\hline
\end{tabular}

the GPE-modified sample. The $\mathrm{C}-\mathrm{O} \sigma$-bond also shows up at about $7 \mathrm{eV}$ in bare chitosan, but is not present in the modified samples. This feature in the modified curves is possibly due to "quenching" as a result of surface roughness and the small penetration depth in the VUV. The surface roughness is on the order of 20-30 nm and the penetration depth is on the order of $36 \mathrm{~nm}$, as determined from the material dielectric function at 7 $\mathrm{eV}$. This means that the path length of light is on the same order $(40-60 \mathrm{~nm})$ as the penetration depth $(36 \mathrm{~nm})$ and we resolve less information about the chitosan underneath the rough surface and chemically modified layer.

$\sigma$-Bond chromophores show the result of adsorption due to $\sigma$-to- $\sigma^{*}$ excitations. $\pi$-Bond chromophores, likewise, show the result of $\pi$-to- $\pi^{*}$ excitations. It is important to note that the energy assignments are approximations because values are taken from tabulated data obtained through the study of compounds in the gaseous or liquid state and not from the solid state. ${ }^{17}$

Second-derivative spectra are useful in oscillator analysis. Figure 9 shows the second derivative of the imaginary part

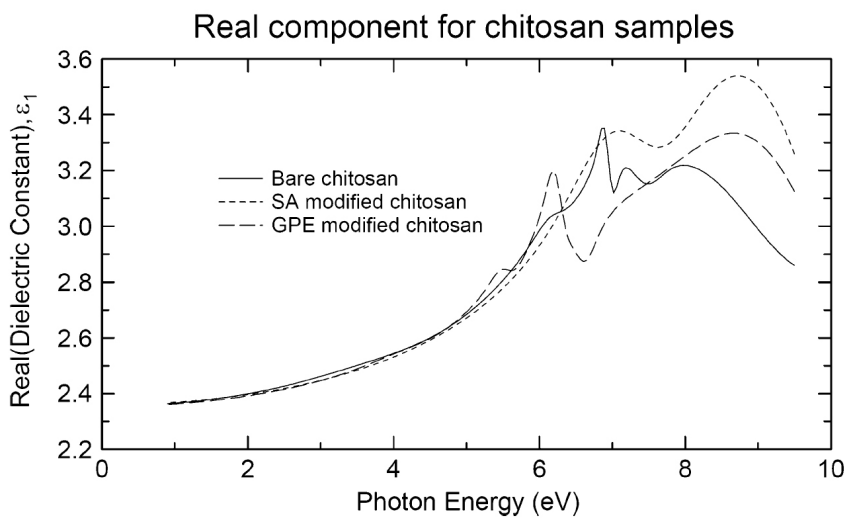

Figure 7. Real part of the dielectric function for bare, GPE-modified, and SA-modified chitosan in the VUV.

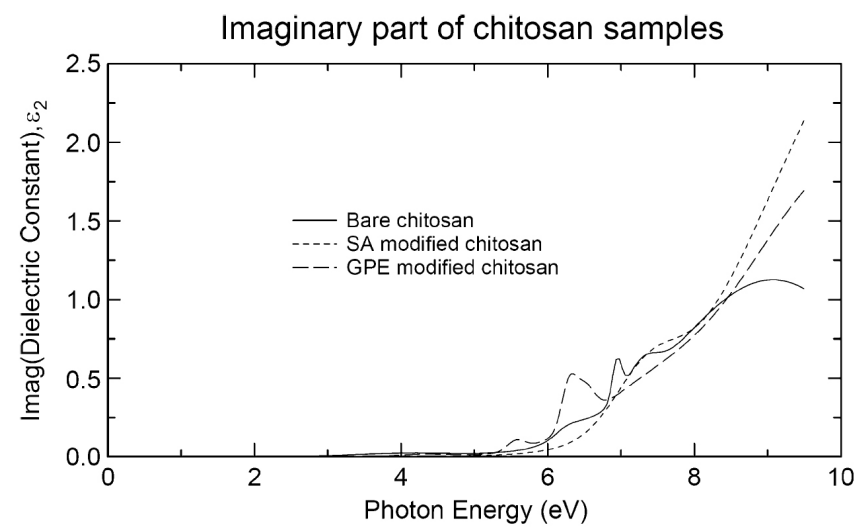

Figure 8. Imaginary part of the dielectric function for bare, GPEmodified, and SA-modified chitosan in the VUV.

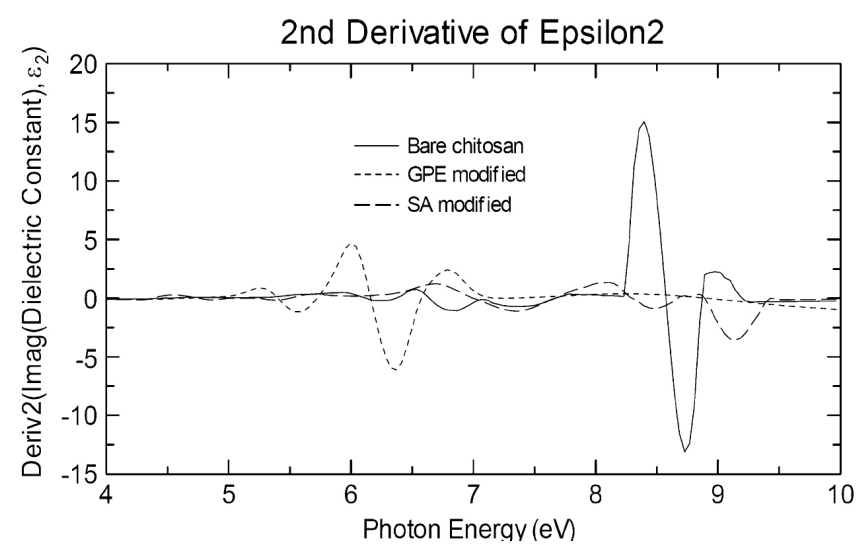

Figure 9. Second derivative of the imaginary part of the dielectric function for bare, GPE-modified, and SA-modified chitosan in the VUV.

of the dielectric function, $\varepsilon_{2}$. Second-derivative spectra show inflection points in the data and help determine the true center energies for oscillators and glean more information about each oscillator. Note the asymmetry in Figure 9 due to some form of nonideality in the Gaussian line shapes, for example, line shape distortions due to optical transitions at photon energies above our experimental range. 


\section{DISCUSSION AND CONCLUSIONS}

Optical constants from near infrared to VUV were determined for unmodified, GPE-and SA-modified, and protein-coated GPE-modified chitosan films. The absorptions due to electronic band transitions were modeled utilizing Gaussian and TaucLorentz oscillator line shapes, and were used to identify numerous surface chemistries.

Well-understood Gaussian line shapes work well for describing these anisotropic and spectrally dependent optical constants. Several resonant structures were observed in the VUV, which influence line shapes at lower photon energy. Apparently large absorptions centered above $10 \mathrm{eV}$ (outside our range) were found for all films. Several resonant oscillator structures exhibit greatly differing oscillator parameters for asdeposited, as well as SA- and GPE-modified films. These inplane and out-of-plane oscillator strengths, energy positions, and broadenings were determined and tabulated. A phenyl resonant $\pi$-bond at approximately $6.3 \mathrm{eV}$ was observed for the GPE-modified sample. This was not present in the bare chitosan data. GPE contains a phenyl group in the molecule and absorption due to it is clearly identified.

VUV-SE is a relatively new technique, and shows promise in being more sensitive to surface chemistry of solids than pure VUV reflectance or visible or infrared spectroscopic ellipsometry. ${ }^{5}$ Until a few years ago VUV optical constants of solids could be determined using only pure reflectance, for which surface sensitivity was minimal. Electron energy loss spectroscopy was available, but the beam damaged the surface and a Kramers-Kronig analysis (which has strong limitations) was necessary. Infrared spectroscopy is a wellestablished technique to determine chemistry; however, it has a high penetration depth and lower surface sensitivity. VUV is higher in photon energy, has shorter penetration depth and high surface sensitivity. For an extinction coefficient value of 0.2 , infrared photons at a wavenumber of $1200 \mathrm{~cm}^{-1}$ can penetrate about $1800 \mathrm{~nm}$ into the material. VUV photons with a wavelength of $180 \mathrm{~nm}$ penetrate $36 \mathrm{~nm}$.

\section{Acknowledgements}

We wish to thank the Army Research Laboratory (contract number W911NF-04-2-0011) for financial support and the College of Engineering and Technology at the University of Nebraska-Lincoln. Thanks are also due to James Hilfiker of the J. A. Woollam Company for acquiring the vacuum ultraviolet ex situ data used in this study.

\section{REFERENCES}

1. Dumitriu, S. Polymeric Biomaterials (2nd ed.). Marcel Dekker: New York, 2002.

2. Nosal, W. H., Thompson, D. W., Yan, L., Sarkar, S., Subramanian, A., Woollam, J. A. Colloids Surf., B 2005; 43: 131.

3. Nosal, W. H., Thompson, D. W., Yan, L., Sarkar, S., Subramanian, A., Woollam, J. A. Colloids Surf., B 2005; 46: 26.

4. Sashiwa, H., Aiba, S. Prog. Polym. Sci. 2004; 29: 887.

5. Hilfiker, J. VUV ellipsometry. In Handbook of Ellipsometry, Tompkins, H. G., Irene, E. A. (eds). William Andrew: 2005; 721.

6. Schubert, M. Thin Solid Films 1998; 313-314: 323.

7. Elman, J. F., Greener, J., Herzinger, C. M., Johs, B. Thin Solid Films 1998; 313-314: 814.

8. Schubert, M. Generalized ellipsometry. In Handbook of Ellipsometry, Tompkins, H. G., Irene, E. A. (eds). William Andrew: 2005; 637.

9. Tompkins, H. G., McGahan, W. A. Spectroscopic Ellipsometry and Reflectometry: A User's Guide. Wiley: New York, 1999.

10. Woollam, J.A., Johs, B., Herzinger, C.M., Hilfiker, J., Synowicki, R., Bungay, C.L. Crit. Rev.Opt. Sci. Technol. 1999; CR72:3.

11. Johs, B., Woollam, J. A., Herzinger, C. M., Hilfiker, J., Synowicki, R., Bungay, C.L. Crit. Rev. Opt. Sci. Technol. 1999; CR72: 29.

12. Born, M., Wolf, E. Principles of Optics 5th ed. Pergamon Press: Oxford, 1975.

13. Bungay, C. L., Tiwald, T. E. Thin Solid Films 2004; 455-456: 272.

14. Meneses, D. D., Malki, M., Echegut, P. J. Non-Cryst. Solids 2006; 351(2): 769.

15. Jellison, G. E., Modine, F. A., Appl. Phys. Lett. 1996; 69: 371; Erratum Appl. Phys. Lett 1996; 69: 2137.

16. Wooten, F. Optical Properties of Solids. Academic Press: New York, 1972.

17. Pretsch, E., Seibl, J., Clerc, T. Tables of Spectral Data for Determination of Organic Compounds (2nd ed.). Springer-Verlag: Berlin, 1989. 